\title{
Pupil Reactions to Tactile Stimulation: A Systematic Review
}

\author{
Mariana de Mello Gusso*, Gabriele Serur and Percy Nohama \\ Laboratório de Engenharia de Reabilitação, Programa de Pós-Graduação em Tecnologia em Saúde, Escola Politécnica, \\ Pontifícia Universidade Católica Do Paraná, Curitiba, Brazil
}

\section{OPEN ACCESS}

Edited by:

Claus-Christian Carbon, University of Bamberg, Germany

Reviewed by:

Angel Nunez,

Autonomous University of

Madrid, Spain

Yong Gu,

Chinese Academy of Sciences

(CAS), China

Stefan Josef Breitschaft,

$B M W$, Germany

*Correspondence:

Mariana de Mello Gusso

ma_gusso@hotmail.com

Specialty section: This article was submitted to

Perception Science,

a section of the journal

Frontiers in Neuroscience

Received: 27 September 2020

Accepted: 12 January 2021

Published: 22 February 2021

Citation:

Gusso MdM, Serur G and Nohama P (2021) Pupil Reactions to Tactile Stimulation: A Systematic Review.

Front. Neurosci. 15:610841. doi: 10.3389/fnins.2021.610841
Pupil dynamics can represent an indirect measure of perception; thus, it has been broadly explored in the auditory and visual fields. Although it is crucial for experiencing the outside world, tactile perception is not well-explored. Considering that, we sought to answer the following question via a systematic review: does normal tactile perception processing modulate pupil dilation in mammals (human or not)? The review process was conducted according to PRISMA Statement. We searched on Periódicos CAPES (Brazil) for the following terms: [(touch) OR (cutaneous stimulation) OR (tactile perception) OR (somatosensory) AND (pupil OR pupillary) NOT blind NOT reflex NOT pain NOT fear NOT noxious NOT autism NOT nerve NOT (pupillary block) NOT glaucoma NOT cataract NOT aneurysm NOT syndrome NOT treatment NOT special education]. From the 6,488 papers found, 4,568 were duplicates, and nine fulfilled the inclusion criteria. All papers found a positive relationship between pupil diameter and tactile perception. We found that the pupil is a reliable indirect measure of brain states and can evaluate norepinephrine (NE)/locus coeruleus (LC) action, stimulus inhibition, arousal, cognitive processes, and affection independently of the stimuli category (visual, auditory, or tactile). We also found that the perceptual tactile processing occurs in similar ways as the other perceptual modalities. We verified that more studies should be done, mostly avoiding low sampling rate recording systems, confounders as cue signs, not automated stimulation, and concurrent stimulus and using more reliable equipment.

Keywords: pupil dilation, tactile stimulation, perception, eye-tracking, norepinephrine system

\section{INTRODUCTION}

To understand the neural basis of consciousness-one of the main goals of modern science (Miller, 2005; Michel et al., 2019) - it is necessary to approach perception in its different modalities. The visual (mainly) and auditory domains have been broadly investigated (Del Cul et al., 2007; Wyart and Tallon-Baudry, 2008; Gaillard et al., 2009; Carbon and Jakesch, 2012; Li et al., 2014; Pitts et al., 2014; Carbon, 2016; Herman et al., 2019). However, there is a lack of study on tactile perception. Proof of this disparity is when comparing searches on the three fields. The search on Periódicos CAPES (a search engine that searches on several databases like Web of Science, PubMed, Elsevier, and OneFile), from the 163,356 papers found on "conscious perception," $35.24 \%$ are on visual, $10.85 \%$ on auditory, and only $7.56 \%$ on tactile/somatosensory conscious perception. Carbon (2016) and Carbon and Jakesch (2012) state that visual dimension dominates the comprehension of the perceptual phenomena and they urge the scientific field to pay more attention to tactile/haptic perception and broaden the understanding of perception. 
Some of the methods of studying the nervous system are functional magnetic resonance imaging (fMRI), scalp electroencephalography (sEEG), magnetoencephalography (MEG), intracranial EEG (icEEG), and pupillometry. fMRI can produce whole-brain images showing contrasts. However, it does not have a high temporal resolution. sEEG and MEG have a higher temporal resolution but are affected by external artifacts and have a low spatial resolution, not accessing deep structures. The method with both good spatial and temporal resolution is icEEG since it records directly from the brain, but it is hard to conduct with humans since it must be done during necessary surgical electrode implant (Herman et al., 2019). Pupillometry is a non-invasive and complementary measure of cognition with a high temporal resolution (Luna et al., 2008; Tatler et al., 2014; Eckstein et al., 2017) that can be an indicator of perception and so a tool for developing no-report paradigms that should avoid the response confounder (Einhauser et al., 2010; Piquado et al., 2010; Laeng and Endestad, 2012; Laeng et al., 2012; Kang and Wheatley, 2015).

Eye metrics are the measurement and evaluation of eye and eyelid dynamics. They provide an ideal and powerful objective measure of ongoing cognitive processes and information requirements during behavior. They are non-invasive, have a high temporal resolution, and are a well-understood neural foundation, providing an ideal neuroscience model to investigate the association between brain mechanisms and behavior (Luna et al., 2008; Tatler et al., 2014; Eckstein et al., 2017). The process of pupil measurement that was once time-consuming is relatively easy to carry out and non-invasive today, having a spatial resolution (in diameter) better than $0.025 \mathrm{~mm}$ on individual measurements at sampling rates of $25-2,000 \mathrm{~Hz}$ (Granholm and Steinhauer, 2004; Eckstein et al., 2017) or lower when using webcam-based systems (Holmqvist et al., 2011; Schriver et al., 2018, 2020).

Pupillometry measures variations in the diameter of the pupillary aperture of the eye in response to physiological or psychological stimuli (Granholm and Steinhauer, 2004; Laeng et al., 2012). Pupil size is changed by two antagonistic muscles: the dilator pupillae and the sphincter pupillae. The sphincter muscle receives input from brain systems involved in pupillary light reflex, and both muscles receive inputs from brain systems involved in cognitive and autonomic functions, being influenced by it (Bremner and Spence, 2017; Eckstein et al., 2017).

Pupil dilation is directly related to conditions of increased attention or cognitive load or of emotional or cognitive arousal. Pupillometry is proven to work as an indicator of perception, language processing, memory and decision making, emotion and cognition, and cognitive development (Sirois and Brisson, 2014).

One explanation for the link between pupil dilation and psychological and physiological stimuli is that the dilation can be attributed to the sympathetic system's activation during autonomic arousal and mental activity, being modulated by the noradrenergic locus coeruleus (LC) (Aminihajibashi et al., 2020). The LC is essential for the regulation of physiological arousal and cognitive functioning. It produces the neurotransmitter norepinephrine (NE) and has direct inhibitory projections to the parasympathetic Edinger-Westphal nucleus, where the pupil's constricting fibers originate, therefore also inhibiting its constriction and indirectly enabling the pupil's dilation. LC also stimulates the sympathetic system, including the fibers that innervate the pupil to dilate it (Sirois and Brisson, 2014; Eckstein et al., 2017).

Pupil study is crucial since it may benefit the evaluation of "special populations who may not be able or willing to provide a typical behavioral answer (complex motor or verbal responses) to certain research questions, such as pre-verbal infants, non-verbal adults, or children with ASD" (autistic spectrum disorder) (Sirois and Brisson, 2014). The close relationship between task-evoked pupil dilation and its underlying neural mechanisms enable the use of this method with participants of any age; knowledge about this relationship allows researchers to relate the neural system and cognitive studies and to interpret results of cognitive studies in terms of underlying neurophysiological processes (Eckstein et al., 2017; Medathati et al., 2020).

Visual and auditory stimuli may cause pupil dilation response both in adults and in infants (Aston-Jones and Cohen, 2005; Einhauser et al., 2010; Piquado et al., 2010; Laeng and Endestad, 2012; Kang and Wheatley, 2015; Wetzel et al., 2016; Eckstein et al., 2017). Moreover, it appears to be affected by arousal rather than attention itself since its reaction to a cue was not a predictor of better performance on a visual task (Aminihajibashi et al., 2020). Pupillary light reflex can be artificially created by showing the sun's pictures, revealing the top-down effects of perception in the pupil dilation. Its properties, like delay, speed, and length of a change in pupil diameter index, affect various aspects of attention and memory (Sirois and Brisson, 2014).

Tactile perception, popularly known as "touch," is the first of our senses to develop (Bremner and Spence, 2017; Miguel et al., 2019). Through the sense of touch, one perceives their own body and develops a sense of self (Carbon and Jakesch, 2012, Bremner and Spence, 2017; Hoffmann et al., 2017; Rigato et al., 2019). Moreover, touch is essential for developing social function (Schneider et al., 2016; Bremner and Spence, 2017; Cascio et al., 2019).

Tactile perception differs from haptic exploration since it does not include movement. The haptic exploration [which encompasses tactile and kinesthetic sense (Lederman and Klatzky, 2009)] gives us access to physical objects and the external world (Hoffmann et al., 2017). Carbon and Jakesch (2012) developed the haptic aesthetic processing model. They hypothesize that the haptic aesthetic experience occurs in three levels. The first is a low level of exploration, the second is a midlevel of assessment, and the last is a high level of evaluation, where the cognitive and emotional processing occurs. There is a feedback loop in each of these levels (expectation, integration, and familiarity, respectively). There are different pupil reactions to these different levels, but further studies need to be done to correlate them to that model.

We rarely use passive tactile perception alone in daily life since we explore the environment to get to know it. Differently from other sensory modalities, the touch is an active process, since what is touched touches back the person who is touching it (Carbon and Jakesch, 2012). In research, if we want to understand the neural correlations of perception, we need to isolate it, 
study touch (lower level processing) and movement separately, avoiding signal noise between the two, and, later, integrate both in the study of haptics. Because of tactile perception's relevance for social and cognitive domains, it should be placed more centrally in the study of perception than it is.

Tactile stimuli can be delivered manually (Van Hooijdonk et al., 2019) or in an automated manner. The most common delivery materials are brushes for affective touch (Loken et al., 2011; Croy et al., 2014; Sailer et al., 2016; Hielscher and Mahar, 2017; Van Hooijdonk et al., 2019), electrical stimulation (Mückschel et al., 2020), or mechanical stimulation that can be delivered by different actuators (Garcia-Hernandez et al., 2014): piezoelectric (Schriver et al., 2018, 2020; Ganea et al., 2020; Lee et al., 2020), pneumatic (Moy et al., 2000; Yoo et al., 2015), electrical motor (Sarakoglou et al., 2012, 2014), ultrasonic (Cugini et al., 2009; Kim et al., 2009; Bordegoni et al., 2010), shape memory alloy (SMA) (Velázquez et al., 2006, 2008; Biet et al., 2008), and micro-actuators based on micro-electro-mechanical systems (MEMS) technologies (Ninomiya et al., 2009, 2011; Sarakoglou et al., 2012, 2014; Streque et al., 2012).

Although pupillometry can be such a powerful tool to indirectly measure cognitive processes and brain activity, the studies relating it to tactile perception are still scarce but relevant. It is essential to know if the findings in other perceptual fields can be translated to tactile perception searching for an integrative perception theory. This way, we present the existing findings that look for clarifying pupil interaction dynamics and tactile stimulation.

\section{METHODS}

Based on the PICOS strategy (Liberati et al., 2009, Figure 1), we sought to answer whether normal tactile perception modulates pupil dilation in mammals (human or not); the review was conducted according to the PRISMA Statement (Liberati et al., 2009; Moher et al., 2009, Figure 2).

To find the papers that would help us answer this question, we used Periódicos CAPES' (https://www.periodicos.capes.gov. $\mathrm{br} /$ ) search engine. This engine searches several databases at the same time. We used the term $\{$ [touch OR (cutaneous stimulation) OR (tactile perception) OR somatosensory] AND (pupil OR pupillary) NOT blind NOT reflex NOT pain NOT fear NOT noxious NOT autism NOT nerve NOT (pupillary block) NOT glaucoma NOT cataract NOT aneurysm NOT syndrome NOT treatment NOT special education $\}$ for this search. We restricted the search to only peer-reviewed full-length papers published until November 2020. After selecting the papers that fitted the selection criteria, we used Google Scholar to find the ones that cited them and included those in the research.

\section{Eligibility Criteria}

From the files found, we selected the ones that were fulllength peer-reviewed papers and were conducted with mammals (human or not) with no medical condition. We excluded the ones that were about noxious stimulation, toxicology, or normal or pathological reflex.

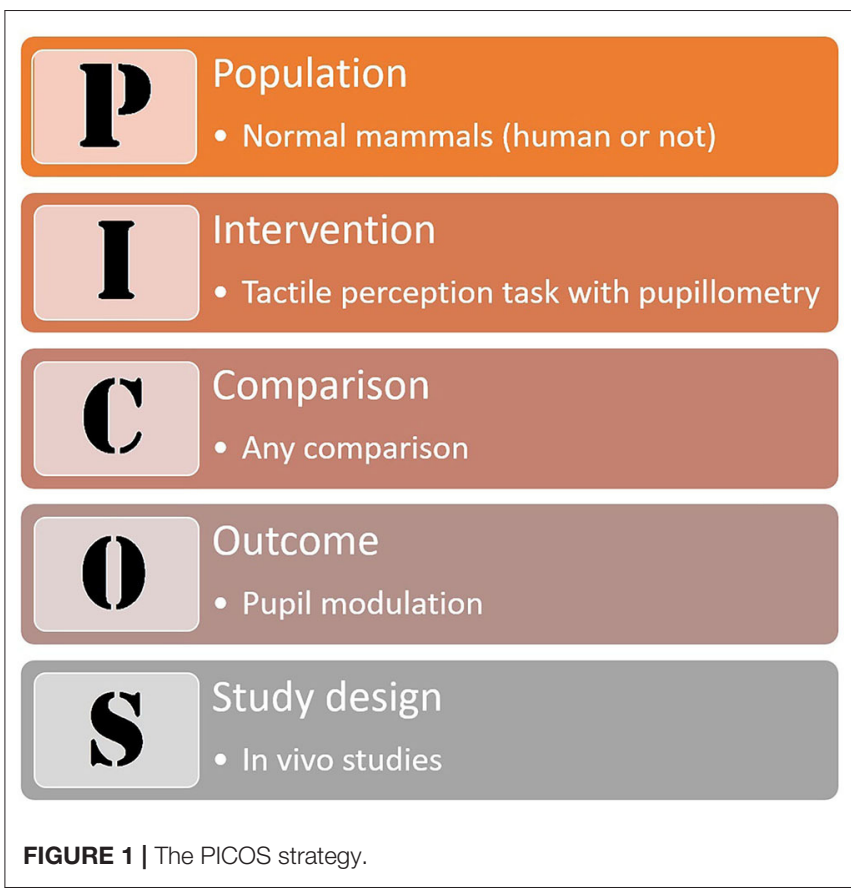

The studies' selection was conducted by two researchers independently that analyzed titles and abstracts and selected papers for full-text appreciation.

\section{Data Collection}

The following information was extracted and tabled from each selected study: (1) authors' names, (2) sample, (3) task design, (4) acquisition system, (5) stimuli, and (6) results.

\section{RESULTS}

\section{Study Selection}

The search engine found papers in 20 databases, as shown in Table 1; from those, eight had papers included in this research. Each paper was indexed in an average of 3.11 of the included databases $(\mathrm{SD}=1.45)$. Science Citation Index Expanded from Web of Science was the database with more papers indexed in; the only paper that was not indexed by them was the one from Ganea et al. (2020).

From the 6,488 papers found, 4,568 were duplicates and nine fulfilled the inclusion criteria, as shown in Figure 2.

\section{Studies' Demographics}

As Table 2 and Figure 3 show, the nine studies were conducted between 1996 and 2020. Three used human subjects (two with adults and one with adolescents) (Van Hooijdonk et al., 2019; Bertheaux et al., 2020; Mückschel et al., 2020), one wild mice (Lee and Margolis, 2016), two with C57BL6J mice (Ganea et al., 2020; Lee et al., 2020), two albino rats (Schriver et al., 2018, 2020), and one juvenile male Japanese monkeys (Macaca fuscata) (Iriki et al., 1996). Four studies used go/no-go tasks (Lee and Margolis, 2016; Schriver et al., 2018, 2020; Mückschel et al., 2020), one a vibration detection task (Lee et al., 2020), one a two-alternative forced choice (2AFC) task (Ganea et al., 2020), one used a passive 


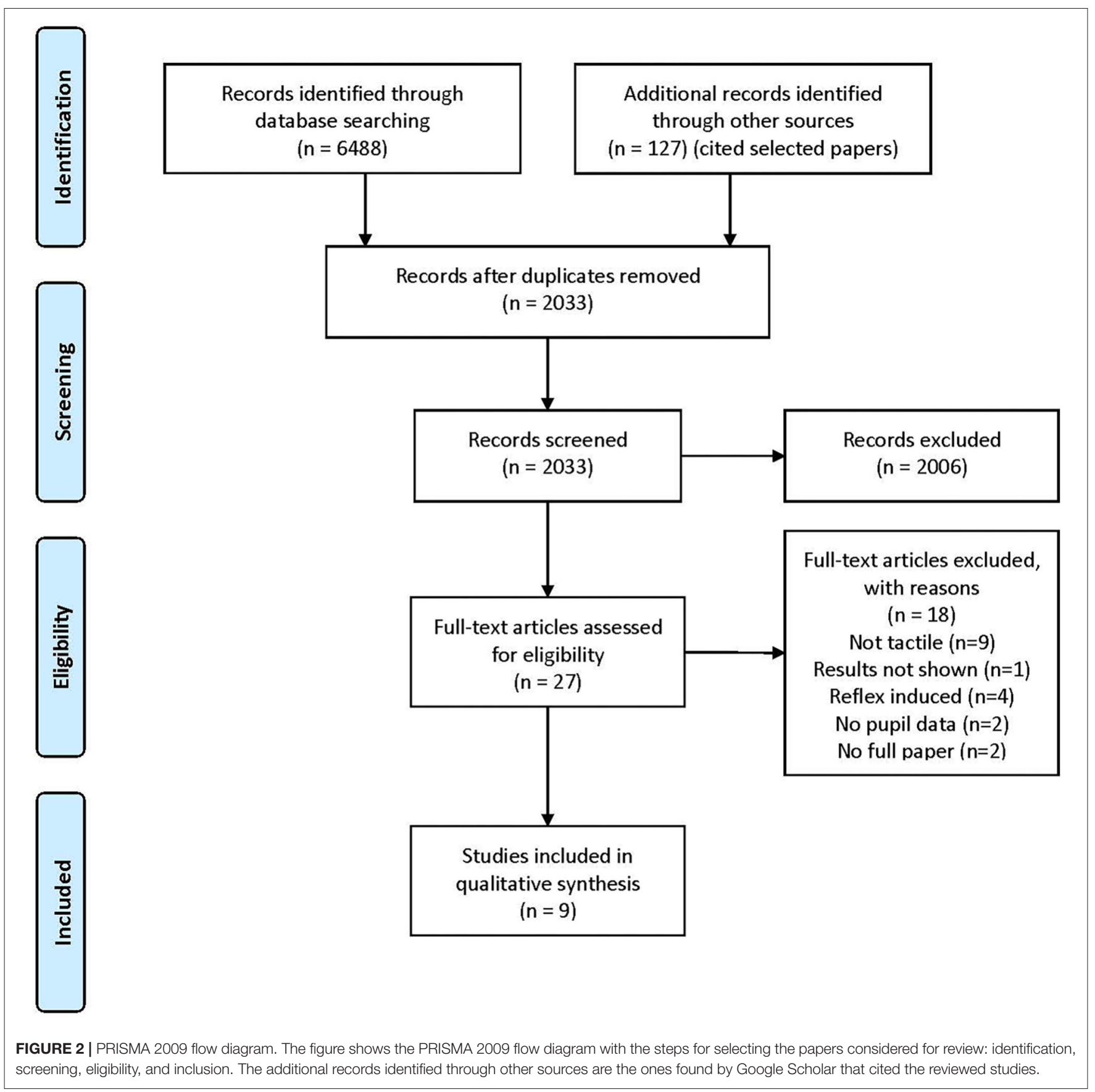

task (Iriki et al., 1996), and two discrimination of affective touch tasks (Van Hooijdonk et al., 2019; Bertheaux et al., 2020).

\section{Pupil Diameter Acquisition Method}

For acquiring the pupil diameter, seven different methods were used (Table 2). Iriki et al. (1996) did not report the equipment other than saying it was a motor-only shot (MOS) camera. The reported equipment were a $10-\mathrm{Hz}$ in-house system (Schriver et al., 2018, 2020), 30-Hz EyeTribe tracker (Van Hooijdonk et al., 2019), Point Gray Chameleon3 camera (Ganea et al., 2020), DMK22BUC03 (Lee et al., 2020), 50-Hz Allied Vision
Technologies Pike F-032 camera (Lee and Margolis, 2016), 60 $\mathrm{Hz}$ ISCAN-ETL-100H (Bertheaux et al., 2020), and $500-\mathrm{Hz}$ RED 500 (Mückschel et al., 2020). All studies had controlled luminance and either excluded the trials where there were blinks or interpolated the data.

\section{Stimulus Sources and Their Technical Characteristics}

Bertheaux et al. (2020) presented 12 different stimulus sources classified as follows: three unpleasant (different sandpaper 
TABLE 1 | Databases found by the search engine.

\begin{tabular}{|c|c|c|}
\hline Database & Papers found & Papers included \\
\hline Scopus (Elsevier) ${ }^{*}$ & 1.478 & 4 \\
\hline OneFile (GALE) ${ }^{\star}$ & 642 & 3 \\
\hline $\begin{array}{l}\text { Science citation index } \\
\text { expanded (Web of Science) }\end{array}$ & 537 & 8 \\
\hline $\begin{array}{l}\text { Taylor \& Francis } \\
\text { Online-Journals }\end{array}$ & 442 & \\
\hline $\begin{array}{l}\text { Technology Research } \\
\text { Database* }\end{array}$ & 385 & 3 \\
\hline $\begin{array}{l}\text { ERIC (U.S. Dept. of } \\
\text { Education) }\end{array}$ & 378 & \\
\hline $\begin{array}{l}\text { Social sciences citation } \\
\text { index (Web of Science) }\end{array}$ & 333 & 2 \\
\hline $\begin{array}{l}\text { Advanced technologies \& } \\
\text { aerospace database }\end{array}$ & 282 & \\
\hline $\begin{array}{l}\text { Engineering research } \\
\text { database }^{\star}\end{array}$ & 280 & 3 \\
\hline SpringerLink & 245 & \\
\hline $\begin{array}{l}\text { Materials science \& } \\
\text { engineering database }\end{array}$ & 235 & \\
\hline $\begin{array}{l}\text { Arts \& humanities citation } \\
\text { index (Web of Science) }\end{array}$ & 184 & \\
\hline PMC (PubMed Central)* & 180 & 2 \\
\hline $\begin{array}{l}\text { Directory of open access } \\
\text { journals }(\mathrm{DOAJ})^{\star}\end{array}$ & 175 & 3 \\
\hline JSTOR archival journals & 157 & \\
\hline $\begin{array}{l}\text { Sage journals (Sage } \\
\text { publications) }\end{array}$ & 148 & \\
\hline $\begin{array}{l}\text { Cambridge journals } \\
\text { (Cambridge University } \\
\text { Press) }\end{array}$ & 135 & \\
\hline Sociological abstracts & 105 & \\
\hline $\begin{array}{l}\text { Computer and information } \\
\text { systems abstracts }\end{array}$ & 103 & \\
\hline Emerald insight & 64 & \\
\hline
\end{tabular}

${ }^{*}$ Databases that had papers included in this review.

roughness levels), seven neutral (three $100 \%$ polylactic material and four mixes of $50 \%$ polylactic material and $50 \%$ other materials), and two pleasant (velvet and synthetic fur). The participants should actively touch the materials with their dominant hand in three rounds of $15 \mathrm{~s}$; the first one was not considered on data analysis to avoid the surprise element. Ganea et al. (2020) delivered different cosine waves to mice whiskers with a piezo bending actuator (Johnson Matthey, Royston, UK) amplified by a piezo controller (MDT693A; Thorlabs, NJ, USA). Schriver et al. (2018) and Schriver et al. (2020) also used a piezoelectric actuator (PL140, Physik Instrumente, Karlsruhe, Germany) driven by a high-voltage amplifier (OPA452; Texas Instruments, Dallas, TX). The 2018 paper used whisker deflections with durations of 25,50, and $100 \mathrm{~ms}$ with respective velocities of $\sim 1,200,600$, and $300^{\circ} / \mathrm{s}$, while they did not report the duration/velocity of the deflections on the 2020 paper. Lee et al. (2020) delivered vibration to the left whisker pad via an aluminum mesh $(2 \times 2 \mathrm{~cm})$ attached to a ceramic piezoelectric wafer (Morgan Matroc, Bedford, $\mathrm{OH}$ ); they delivered a series of discrete Gaussian deflections: 15- vs. $10-\mathrm{ms}$ pause with a frequency of $40 \mathrm{~Hz}$ and a total duration of $300 \mathrm{~ms}$ with five different amplitudes: 0, 10, 20,40, and $80 \mu \mathrm{m}$. Iriki et al. (1996) used a probe to the fingers with a pressure transducer attached to record the stimulation's timing and strength. Lee and Margolis (2016) used 120- and 1,200grid sandpaper. Mückschel et al. (2020) delivered 150 and $40 \mathrm{~Hz}$ sinewave stimulus via a miniature electromagnetic stimulator (Dancer Design; for detailed information, see http://www. dancerdesign.co.uk). Lastly, Van Hooijdonk et al. (2019) used a foundation brush (goat hair; conducted pressure $\sim 11.5 \mathrm{~Pa}$ ). In personal contact with the author, they clarified that the experimenter stroked the participant's forearm with a foundation brush. The timing was controlled by marking a distance on the arm and stroking it in the required time to achieve the velocity of that condition (e.g., 6-cm distance requires stroking of 2 s) (Table 2).

\section{Results of the Selected Studies}

All studies indicated a positive correlation between stimuli perception and pupil dilation (Table 2). As shown in Figure 3, while Bertheaux et al. (2020) found that neutral materials lead to a lower pupil dilation than materials with high emotional intensity, independently of their valence (being pleasant or unpleasant), Van Hooijdonk et al. (2019) associated the difference in the pupil dilation only with the stimulus intensity, not subjective pleasantness. The four go/no-go tasks (Lee and Margolis, 2016; Schriver et al., 2018, 2020; Mückschel et al., 2020) and the 2AFC task (Ganea et al., 2020) found a significantly broader pupil dilation for go trials than no-go ones. Lee and Margolis (2016) and Ganea et al. (2020) also found that not only the correct behavior had a bigger pupil but also the false alarms, associating the pupil dilation with the type of behavioral response rather than the type of stimulus presented. Ganea et al. (2020) and Schriver et al. (2018) showed that the pre-stimulus pupillary size reflected task engagement. Iriki et al. (1996) found that primary sensory area (SI) neurons responded to a light touch of the glabrous skin, two neurons to manipulation of finger joints; during the period, no pupil dilation was induced (monkey was not attentive). When attentional dilation was induced during the task, another 10 neurons responded to the glabrous skin's stimulation. Lee et al. (2020) calculated cross-correlation between pupil diameter and detection performance. It was computed from the average of pupil dilation and behavior in a five-trial sliding window. They found a positive correlation between detection performance and pupil dilation, with pupil diameter lagging behind performance by a median of 9.2 trials. They also found that the pupil dilation's amplitude was positively influenced by the licking, response time, and detection rate.

\section{DISCUSSION}

\section{Pupil Diameter Acquisition Method}

The selected studies' sampling rate range was between 10 and $500 \mathrm{~Hz}$ (Table 3), even with most of them having a small 
TABLE 2 | Overview of included studies.

\begin{tabular}{|c|c|c|c|c|c|}
\hline References & Sample & Task design & Acquisition system & Stimuli & Results \\
\hline Bertheaux et al. (2020) & $\begin{array}{l}25 \text { humans ( } 12 \text { males, } \\
18-27 \text { years old) }\end{array}$ & $\begin{array}{l}\text { Discrimination of } \\
\text { affective touch }\end{array}$ & ISCAN-ETL-100H & 12 affective materials & $\begin{array}{l}\text { Affective touch } \\
\text { modulates pupil. }\end{array}$ \\
\hline Iriki et al. (1996) & $\begin{array}{l}2 \text { Macaca fuscata (2 } \\
\text { males, juvenile) }\end{array}$ & Passive stimulation & $\begin{array}{l}\text { Motor only shot (MOS) } \\
\text { camera }\end{array}$ & $\begin{array}{l}\text { Probe to the fingers } \\
\text { with a pressure } \\
\text { transducer }\end{array}$ & $\begin{array}{l}\text { Pupil dilation onset } \\
\text { occurred before the } \\
\text { somatosensory } \\
\text { stimulus associated } \\
\text { with the clue. }\end{array}$ \\
\hline $\begin{array}{l}\text { Lee and Margolis } \\
\text { (2016) }\end{array}$ & $\begin{array}{l}6 \text { wild mice ( } 5 \text { males, } \\
63-79 \text { days old) }\end{array}$ & Go/no-go task & $\begin{array}{l}\text { Allied Vision } \\
\text { Technologies Pike } \\
\text { F-032 camera }\end{array}$ & $\begin{array}{l}\text { 120- and 1,200-grid } \\
\text { sandpaper }\end{array}$ & $\begin{array}{l}\text { Pupil dilation was } \\
\text { related to response } \\
\text { rather than stimuli. }\end{array}$ \\
\hline Lee et al. (2020) & $\begin{array}{l}7 \text { C57BL/6J mice (7 } \\
\text { males, } 4 \text { weeks old) }\end{array}$ & Vibration detection task & DMK22BUC03 & Piezo-driven mesh & $\begin{array}{l}\text { Pupil dilation at } \\
\text { baseline is related to } \\
\text { detection performance. }\end{array}$ \\
\hline Schriver et al. (2018) & $\begin{array}{l}5 \text { albino rats ( } 5 \text { females, } \\
6-10 \text { months old }{ }^{\star} \text { ) }\end{array}$ & Go/no-go task & $\begin{array}{l}\text { In-house system } \\
\text { (FL3-U3-13Y3M-C; } \\
\text { FLIR) }\end{array}$ & $\begin{array}{l}\text { Piezoelectric bending } \\
\text { actuator }\end{array}$ & $\begin{array}{l}\text { Pupil dilation at } \\
\text { baseline is related to } \\
\text { detection performance } \\
\text { and reaction time. }\end{array}$ \\
\hline Schriver et al. (2020) & $\begin{array}{l}8 \text { albino rats (8 females, } \\
\left.6-10 \text { months old }{ }^{\star}\right)\end{array}$ & Go/no-go task & $\begin{array}{l}\text { In-house system } \\
\text { (FL3-U3-13Y3M-C; } \\
\text { FLIR) }\end{array}$ & $\begin{array}{l}\text { Piezoelectric bending } \\
\text { actuator }\end{array}$ & $\begin{array}{l}\text { Pupil dilation is } \\
\text { influenced by stimulus } \\
\text { encoding and decision } \\
\text { formation. }\end{array}$ \\
\hline $\begin{array}{l}\text { Van Hooijdonk et al. } \\
\text { (2019) }\end{array}$ & $\begin{array}{l}28 \text { human adults ( } 11 \\
\text { males, } 19.14 \pm 1.02 \\
\text { years old) }\end{array}$ & $\begin{array}{l}\text { Discrimination of } \\
\text { affective touch }\end{array}$ & EyeTribe tracker & Foundation brush & $\begin{array}{l}\text { Touch-induced pupil } \\
\text { size reflects stimulus } \\
\text { intensity. }\end{array}$ \\
\hline
\end{tabular}

*Information obtained via e-mail with the paper's contact author.

sampling rate-modern eye-trackers can achieve 2,000 $\mathrm{Hz}-$ they have proved that pupil dynamics can be seen as either an impoverished measure of brain function or a rich measure of cognition as Eckstein et al. (2017) has previously defended. While the $30-\mathrm{Hz}$ systems (Van Hooijdonk et al., 2019; Ganea et al., 2020; Lee et al., 2020) are the slowest commercially available, there was an in-house-developed system that was only $10 \mathrm{~Hz}$ (Schriver et al., 2018, 2020). Typically used are 50 and $60 \mathrm{~Hz}$ (Lee and Margolis, 2016; Bertheaux et al., 2020) since these are the most common frequencies in camera technology for a long time. The $500 \mathrm{~Hz}$ (Mückschel et al., 2020) started to be used in 2000 and, from the studies found in this research, is the best sampling rate. The ideal sampling rate, especially when analyzing eye movements, is $>250 \mathrm{~Hz}$; no mathematical method defines a cutoff value, but it instead has been established through consensus (Holmqvist et al., 2011). Since equipment with low sampling rates is cheaper, it is broadly used.

Holmqvist et al. (2011) stated that a low sampling rate could be compensated for by enlarging the number of data acquired in a quadratic manner. This way, data acquired by $10 \mathrm{~Hz}$ (SRa) would need to have 625 times more data to have the same quality as a study at $250 \mathrm{~Hz}$ (the minimum ideal sampling rate-SRi). As shown in the following equation:

$$
x=\left(\frac{\mathrm{SRi}}{\mathrm{SRa}}\right)^{2}
$$

Table 3 shows how many times each dataset would have to be larger than a dataset with a $250-\mathrm{Hz}$ recording and the total number of trials for each study presented here. Four of the works with a small sampling rate had a total number of trials bigger than 10,000 (Schriver et al., 2018, 2020; Ganea et al., 2020; Lee et al., 2020), which should compensate for the low recording sampling rate. Meanwhile, three other studies had fewer trials (Lee and Margolis, 2016; Van Hooijdonk et al., 2019; Bertheaux et al., 2020). Bertheaux et al. (2020) had only 300 trials total, and for Iriki et al. (1996), data were not available. The three studies with fewer trials are still analyzed here, but their results should be taken carefully and validated through coherence with the other studies.

\section{Stimulus}

The automated systems, as in the four go/no-go, the vibration detection task, and the 2AFC tasks (Lee and Margolis, 2016; Schriver et al., 2018, 2020; Ganea et al., 2020; Lee et al., 2020; 


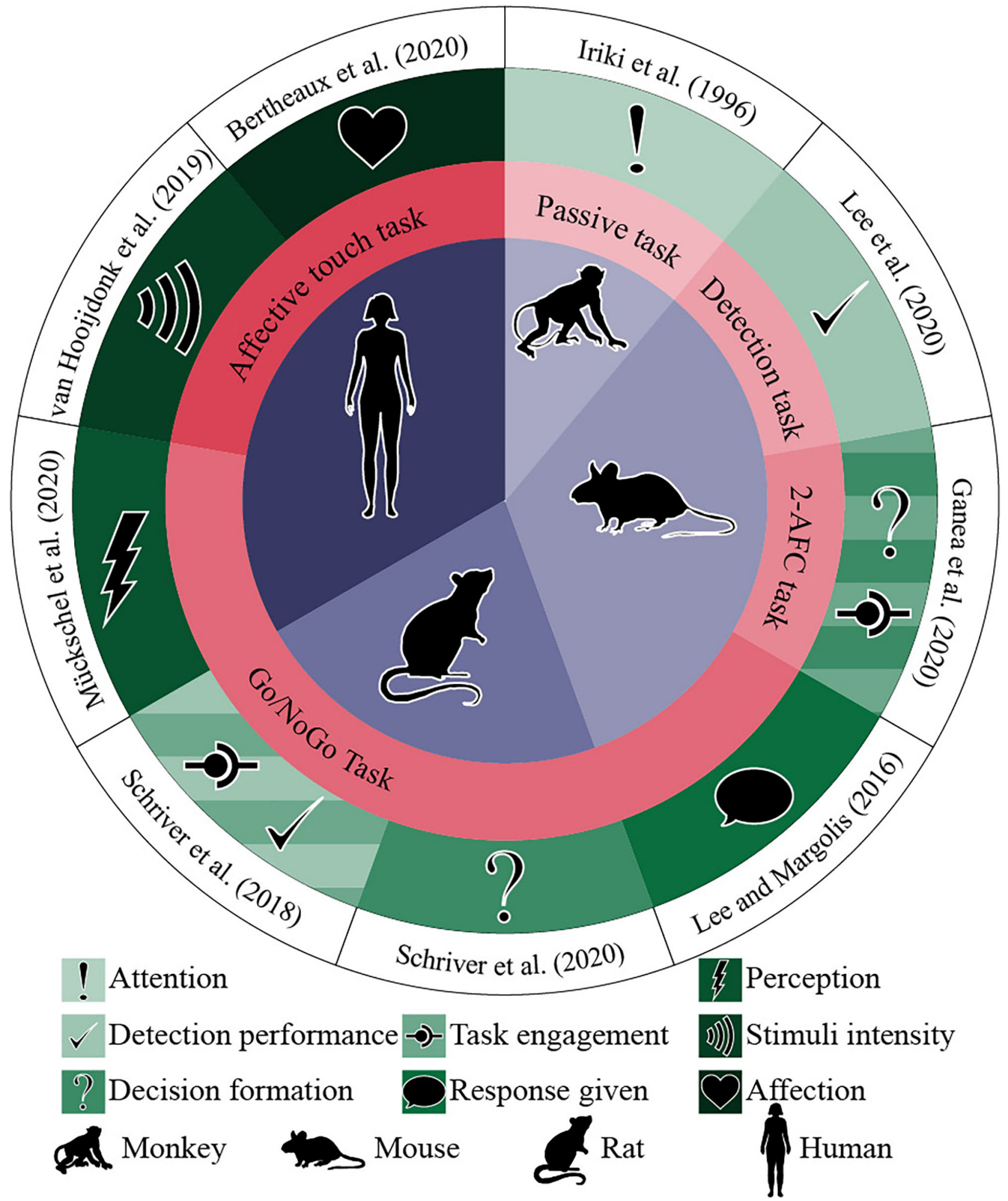

FIGURE 3 | Studies, participants, task type, and main results. The circles from the center outwards represent (1) research participants (purple), (2) task type (coral), (3) positive correlation with pupil dilation (green), and (4) paper's authors and year (white). 
TABLE 3 | Number of times each dataset would have to be larger than a dataset with a 250-Hz sampling rate.

\begin{tabular}{|c|c|c|c|c|c|c|}
\hline References & SR (Hz) & Ideal & $N$ & $t / s$ & $s$ & Total \\
\hline Bertheaux et al. (2020) & 60 & 17.36 & 25 & 12 & 1 & 300 \\
\hline Ganea et al. (2020) & 30 & 69.45 & 8 & 336.2 & 11.5 & 30,930 \\
\hline Iriki et al. (1996) & 14.29 & 204 & 2 & $350 ?$ & $\mathrm{~N} / \mathrm{A}$ & $\mathrm{N} / \mathrm{A}$ \\
\hline Lee and Margolis (2016) & 50 & 25 & 6 & $\sim 106$ & $\sim 4$ & 2,766 \\
\hline Lee et al. (2020) & 30 & 69.45 & 7 & $300-400$ & $5^{\star}$ & $\sim 13,400^{\star}$ \\
\hline Mückschel et al. (2020) & 500 & 0 & 22 & 208 & 4 & 18,304 \\
\hline Schriver et al. (2018) & 10 & 625 & 5 & $\sim 344$ & $\sim 22$ & 38,249 \\
\hline Schriver et al. (2020) & 10 & 625 & 8 & $\sim 300^{*}$ & $\sim 24$ & $\sim 57,000^{\star}$ \\
\hline Van Hooijdonk et al. (2019) & 30 & 69.45 & 28 & 2 & 27 & 1,512 \\
\hline
\end{tabular}

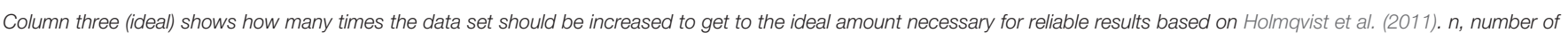

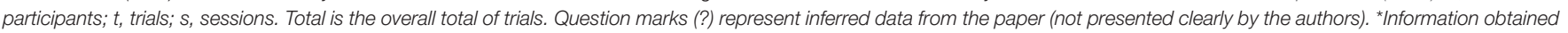
via e-mail with the paper's contact author.

Mückschel et al., 2020) for stimuli deliverance are the most reliable since they depend neither on the participants' interaction with the object (Bertheaux et al., 2020) nor the researcher presenting the stimulus (Iriki et al., 1996; Van Hooijdonk et al., 2019). Participant's interaction may be a confounder since the researchers cannot dissociate the tactile perception from movement results. The studies on which the researcher was responsible for presenting the stimuli could lead to human flaws that Iriki et al. (1996) accounted for when they added a pressure transducer to the stimulation's strength and timing, but not reported by Van Hooijdonk et al. (2019). The range of stimuli in the studies and the lack of automated systems reflect the reality of the field and the fact that there is less research relating tactile perception to pupil dilation than visual or auditory (Aston-Jones and Cohen, 2005; Einhauser et al., 2010; Piquado et al., 2010; Laeng and Endestad, 2012; Kang and Wheatley, 2015; Wetzel et al., 2016; Eckstein et al., 2017)—-tactile stimuli are harder to deliver and to monitor.

\section{Aims and Results of the Selected Studies}

Although all papers linked pupil dilation and tactile perception, they had different aims, and from those aims came different interpretations of the results, showing the multifaceted character of pupil-related research.

Bertheaux et al. (2020) and Van Hooijdonk et al. (2019) tried to find the relation between pupil dilation and pleasantness of the stimulus. Both found that dilation is related to perception, but they had different approaches and results interpretation. Bertheaux et al. (2020) found that when exploring different materials-not considering the first pupil dilation related to the surprise of the touch-the pupil was modulated by the affect. As in auditory and visual studies, both pleasant and unpleasant stimuli led to larger pupil dilation than neutral ones (Partala and Surakka, 2003; Bradley et al., 2008). Van Hooijdonk et al. (2019) used a foundation brush to deliver stimulus for $15 \mathrm{~s}$ in different velocities, considering that the mean velocity $(3 \mathrm{~cm}$ $\mathrm{s}^{-1}$ ) was considered a pleasant one compared to two other neutral velocities $\left(0.3\right.$ and $\left.30 \mathrm{~cm} \mathrm{~s}^{-1}\right)$. They analyzed data from the moment the brush touched the skin and found that pupil dilation was influenced by the stroke's velocity rather than its pleasantness. Opposite to Bertheaux et al. (2020), they state that any sympathetic response to affective touch is probably too small, leading to it being overshadowed by the sympathetic response due to increasing the amount of A-beta tactile input (touch itself). From these two studies, we can hypothesize that the relationship with the stimulus' pleasantness can be divided into two moments: a first moment of arousal, when pupil dilation is related to the stimulus' strength, and a second moment of exploration, when it is emotionally modulated-also asserted by Bradley et al. (2008). Studies from the visual and auditory fields relate pupil dilation with affective stimulus (Partala and Surakka, 2003; Bradley et al., 2008), but those two tactile studies are still incomplete. Further studies associating pleasantness of a stimulus and pupil dilation should be developed. Unlike the two here reported, they should automate the yielded stimuli, recording with a higher sampling rate and a larger amount of trials, and integrate both early and later pupillary reactions to verify the hypothesis formulated here.

Iriki et al. (1996) used the pupil as an objective indicator of attention. Although they did not analyze the effect of the tactile stimuli on the pupil, they found that when the pupil dilation indicated attention, more SI neurons responded to the stimulation of the glabrous skin. They defend that the somatosensory cortex is an active processor that controls the perceptual processes depending on the current behavioral context. In a similar interpretation of data, Schriver et al. (2018) found that the pupil baseline positively correlated to the behavior outcome; they determined that the sensitivity peak was achieved with intermediate pupil baselines. These results are consistent with a visual task that found that tonic arousal influenced early target selection signals and decision formation consistency; in contrast, phasic arousal affected behavior through its relation to attentional engagement and decision formation consistency (Van Kempen et al., 2019). A speech perception study (Ayasse and Wingfield, 2019) stated that the baseline pupil reflects a heightened arousal level in poorer-hearing participants that dissipated over trials. They suggest that the baseline pupillary response may also be connected with the effort necessary or expected related to the task and not only a single construct. 
Even with different aims and results, the four go/no-go and the 2AFC tasks considered the importance of the norepinephrine (NE) and locus coeruleus (LC) on the pupil dilation process. Lee and Margolis (2016) and Ganea et al. (2020) aimed to determine the pupil dynamics association with task performance and found out that pupil dilation was present both on hit and false alarm. They correlated the increase in dilation with the sensory processing and preparation for action, and they were able to predict the choice with $80 \%$ precision. Ganea et al. (2020) also found that pre-stimulus pupillary size reflected mice engagement on the task; they were able to predict if mice would report perceiving the stimulus for both hit and false alarm, which is congruent with Aminihajibashi et al.'s (2020) findings in a visual task, with which they concluded that the pupil appears to be affected by arousal, rather than attention itself, since its reaction to a cue was not a predictor of better performance. Interestingly, Schriver et al. (2018) found biexponential curve shapes for pupil dilation similar to the one elicited by phasic LC activation by Liu et al. (2017) and Joshi et al. (2016) for the hit, miss, and correct reject trials, but not for false alarm ones, which showed a plateau pattern that still needs investigation for understanding its cause.

A not-yet-published work by Yang et al. (2020) simultaneously measured the LC and cortical activity and pupil diameter during a go/no-go single whisker detection task. They found that the LC spiking activity and pupil dilation were related during all the trials. As hypothesized by the studies presented here (Lee and Margolis, 2016; Schriver et al., 2018, 2020; Ganea et al., 2020), they also found a spike on LC aligned to "go" responses (hits and false alarm trials). Another finding was spiking related to the tone that indicated the beginning of a new trial; this activity was lower for hits than misses, with the pupil diameter being larger for the latter on the baseline period. These findings are similar to Iriki et al. (1996) but differ from Schriver et al. (2018) that found that intermediated pupil baselines corresponded to perceptual sensitivity peak in a quadratic/non-linear manner. They also found that LC spikes preceded SI depolarizations and pupil dilations. LC's spiking correlated with both Vm and pupil diameter changes, but on vastly different timescales, they also showed that the time derivative of pupil diameter, but not the absolute pupil size, is a good predictor of SI Vm fluctuations (Yang et al., 2020).

Instead of considering the "go" trials, Mückschel et al. (2020) emphasized the inhibition process of the "no-go" trial. They found that for adolescents - who typically express more difficulty inhibiting SI stimuli (Bodmer et al., 2018) — there was a positive correlation between pupil size and SI inhibition. While Lee and Margolis (2016) associated their findings with the engagement of arousal systems as NE LC neurons linked to onset cues, Ganea et al. (2020) associated their findings with LC-NE adaptive gain theory, and Mückschel et al. (2020) affirm that the NE system is not well-inhibited by lower-level processing of SI stimulation in adolescents.

\section{Equivocated Results Interpretation}

Scientists should not jump to conclusions based on their judgment. The Universal Declaration on Bioethics and Human Rights states that "no individual or group should be discriminated against or stigmatized on any grounds, in violation of human dignity, human rights and fundamental freedoms" (Unesco, 2005). Nonetheless, Bertheaux et al. (2020) brought two biased interpretations. Two of their participants considered synthetic animal fur unpleasant, and they interpreted that it was because the participants grew up in countries where animals are dangerous. They inferred this from their nationality with no recorded inquisition about why they considered the fur unpleasant. They did not consider factors as familiarity, fluency, typicality, and complexity that Carbon and Jakesch (2012) listed as relevant for emotional and cognitive haptic processing. They also found differences in pupil dilation between men and women, which they attributed to the fact that "sandpaper is mainly used by men in construction; manufacturing and its surface appearance may seem more familiar to them" (p. 11). There is evidence that men and women have different pupil dilation patterns, not necessarily associated with the kind of tactile stimulation received; those patterns can predict gender with $90 \%$ accuracy (Costa-Abreu et al., 2015) - this study was not included in this revision since it is only an abstract.

\section{Future Perspectives}

More studies correlating tactile stimulation and pupillometry should be done, mostly avoiding the confounders as cue signs, not automated stimulation, low recording sampling rate, and concurrent stimulus. We suggest using threshold tasks, already explored in vision (Pins and Ffytche, 2003; Ress and Heeger, 2003; Wyart and Tallon-Baudry, 2008; Herman et al., 2019) and audition (Colder and Tanenbaum, 1999; Christison-Lagay et al., 2018).

Due to the importance of developing self and improving social relations (Bremner and Spence, 2017), the study of affective touch still has a long way to go. One of the major problems found in the study of Van Hooijdonk et al. (2019) was that the stimuli were delivered manually with a foundation brush. The same kind of stimuli has already been delivered using robotic systems to ensure its precision (Loken et al., 2011; Croy et al., 2014; Sailer et al., 2016; Hielscher and Mahar, 2017) and should also be applied in the pupillometric research.

The go/no-go studies seem to be a robust and reliable tool for the study of tactile perception. Nonetheless, most of the studies presented here used low sampling rate recording systems (Lee and Margolis, 2016; Schriver et al., 2018, 2020). The acquisition method should be more similar to Mückschel et al. (2020) (sampling rate $\geq 250 \mathrm{~Hz}$ ), but with a more in-depth analysis of the variances, especially at the baseline period as in Schriver et al. (2018), and taking into account the multi-factor process of perception and report (e.g., stimulus encoding and decision formation) as in Schriver et al. (2020).

Based on the studies found here and the ones from the visual and auditory field, pupillometry can be an indirect indicator of cognitive processes. Since pupil dynamics seem to represent a potentially powerful, relatively inexpensivebut still reliable and robust-tool for gauging perceptual and 
cognitive processing in the absence of an overt perceptual report, it leverages these metrics in the development of noreport paradigms in future studies across sensory modalities. Such studies would allow the research of consciousness in a more "pure" way since it will not have confounder facts as the activity of memorizing the stimulus and getting ready to answer a question (Colder and Tanenbaum, 1999; Pins and Ffytche, 2003; Ress and Heeger, 2003; Luna et al., 2008; Einhauser et al., 2010; Piquado et al., 2010; Laeng and Endestad, 2012; Tatler et al., 2014; Kang and Wheatley, 2015; Eckstein et al., 2017; ChristisonLagay et al., 2018; Aminihajibashi et al., 2020; Ganea et al., 2020).

For confirmation of the information found here, we suggest reproducing the low sampling rate studies with a higher one (Lee and Margolis, 2016; Schriver et al., 2018, 2020; Van Hooijdonk et al., 2019; Bertheaux et al., 2020; Ganea et al., 2020; Lee et al., 2020). Also, since Lee and Margolis (2016) and Ganea et al. (2020) found that the pupil can reliably predict behavior, more studies should be done with no-report tasks.

We also propose more research in the different steps of haptic processing, not only on lower levels but also on mid and highlevel processing, as discussed by Carbon and Jakesch (2012).

\section{CONCLUSION}

The assessed literature confirms that pupil dilation is a reliable indirect measure of brain states and can evaluate LC/NE action, stimulus inhibition, arousal, cognitive processes, and affection. During a tactile task, pupil dilation reflects both tonic and phasic changes on the LC that can be associated with the perception itself, independently of

\section{REFERENCES}

Aminihajibashi, S., Hagen, T., Laeng, B., and Espeseth, T. (2020). Pupillary and behavioral markers of alerting and orienting: An individual difference approach. Brain Cogn. 143:105597. doi: 10.1016/j.bandc.2020.105597

Aston-Jones, G., and Cohen, J. D. (2005). Adaptive gain and the role of the locus coeruleus-norepinephrine system in optimal performance. J. Compar. Neurol. 493, 99-110. doi: 10.1002/cne.20723

Ayasse, N. D., and Wingfield, A. (2019). Anticipatory baseline pupil diameter is sensitive to differences in hearing thresholds. Front. Psychol. 10:2947. doi: 10.3389/fpsyg.2019.02947

Bertheaux, C., Toscano, R., Fortunier, R., Roux, J. C., Charier, D., and Borg, C. (2020). Emotion measurements through the touch of materials surfaces. Front. Hum. Neurosci. 13:455. doi: 10.3389/fnhum.2019.00455

Biet, M., Casiez, G., Giraud, F., and Lemaire-Semail, B. (2008). "Discrimination of virtual square gratings by dynamic touch on friction based tactile displays," in 2008 Symposium on Haptic Interfaces for Virtual Environment and Teleoperator Systems, eds J. Weisenberger, A. Okamura and K. Maclean (Reno, NV: IEEE), 41-48. doi: 10.1109/HAPTICS.2008.4479912

Bodmer, B., Friedrich, J., Roessner, V., and Beste, C. (2018). Differences in response inhibition processes between adolescents and adults are modulated by sensory processes. Dev. Cogn. Neurosci. 31, 35-45. doi: 10.1016/j.dcn.2018.04.008

Bordegoni, M., Cugini, U., Covarrubias, M., and Antolini, M. (2010). "A force and touch sensitive self-deformable haptic strip for exploration and deformation of digital surfaces," in International Conference on Human Haptic Sensing and Touch Enabled Computer Applications (Amsterdam: Springer), 65-72. doi: 10.1007/978-3-642-14075-4_10 the stimuli's nature (Colder and Tanenbaum, 1999; Pins and Ffytche, 2003; Ress and Heeger, 2003; Wyart and Tallon-Baudry, 2008; Christison-Lagay et al., 2018; Herman et al., 2019) More studies are required to confirm these investigations' results (as some have been done with relatively low sampling rates).

\section{DATA AVAILABILITY STATEMENT}

The original contributions presented in the study are included in the article, further inquiries can be directed to the corresponding author/s.

\section{AUTHOR CONTRIBUTIONS}

MG was responsible for the conception of the review, literature research, and manuscript writing. GS was responsible for literature research and paper review. PN supervised the selection of the studies and contributed to the revision of the manuscript. All authors revised, read, and approved the submitted version.

\section{FUNDING}

This work was supported by the Conselho Nacional de Desenvolvimento Científico e Tecnológico (CNPq) (Grant No 314241/2018-3), the Coordenação de Aperfeiçoamento de Pessoal de Nível Superior (CAPES) (Grant Nos $88887.147295 / 2017-00, \quad 88881.186875 / 2018-01, \quad$ and 88887.486190/2020-00), and the Fundação Araucária and CAPES (Grant No 88887.185226/2018-00).

Bradley, M. M., Miccoli, L., Escrig, M. A., and Lang, P. J. (2008). The pupil as a measure of emotional arousal and autonomic activation. Psychophysiology 45, 602-607. doi: 10.1111/j.1469-8986.2008.00654.x

Bremner, A. J., and Spence, C. (2017). The development of tactile perception. $A d v$. Child Dev. Behav. 52, 227-268. doi: 10.1016/bs.acdb.2016.12.002

Carbon, C.-C. (2016). Beyond the predominance of the visual empire: a functional model on haptics \& more. Electro. Imag. 2016, 1-2. doi: 10.2352/ISSN.2470-1173.2016.16.HVEI-098

Carbon, C.-C., and Jakesch, M. (2012). A model for haptic aesthetic processing and its implications for design. Proc. IEEE 101, 2123-2133. doi: 10.1109/JPROC.2012.2219831

Cascio, C. J., Moore, D., and Mcglone, F. (2019). Social touch and human development. Dev. Cognit. Neurosci. 35, 5-11. doi: 10.1016/j.den.2018.04.009

Christison-Lagay, K. L., Micek, C., Kronemer, S. I., Forman, M., Aksen, M., AbdelAty, A., et al. (2018). "Investigating auditory conscious perception with a threshold task and intracratial EEG," in Neuroscience (San Diego, CA: Society for Neuroscience).

Colder, B. W., and Tanenbaum, L. (1999). Dissociation of fMRI activation and awareness in auditory perception task. Cognit. Brain Res. 8, 177-184. doi: 10.1016/S0926-6410(99)00015-4

Costa-Abreu, M. D., Fairhurst, M., and Erbilek, M. (2015). "Exploring gender prediction from iris biometrics," in 2015 International Conference of the Biometrics Special Interest Group (BIOSIG) (Darmstadt). doi: 10.1109/BIOSIG.2015.7314602

Croy, I., Angelo, S. D., and Olausson, H. (2014). Reduced pleasant touch appraisal in the presence of a disgusting odor. PLoS ONE 9:e92975. doi: 10.1371/journal.pone.0092975 
Cugini, U., Bordegoni, M., Covarrubias, M., and Antolini, M. (2009). "Geodesic haptic device for surface rendering," in EGVE/ICAT/EuroVR (Lyon), 21-28. doi: 10.2312/EGVE/JVRC09/021-028

Del Cul, A., Baillet, S., and Dehaene, S. (2007). Brain dynamics underlying the nonlinear threshold for access to consciousness. PLoS Biol. 5:e260. doi: 10.1371/journal.pbio.0050260

Eckstein, M. K., Guerra-Carrillo, B., Singley, A. T. M., and Bunge, S. A. (2017). Beyond eye gaze: what else can eyetracking reveal about cognition and cognitive development? Dev. Cogn. Neurosci. 25, 69-91. doi: 10.1016/j.dcn.2016.11.001

Einhauser, W., Koch, C., and Carter, O. (2010). Pupil dilation betrays the timing of decisions. Front. Human Neurosci. 4:18. doi: 10.3389/fnhum.2010.00018

Gaillard, R., Dehaene, S., Adam, C., Clémenceau, S., Hasboun, D., Baulac, M., et al. (2009). Converging intracranial markers of conscious access. PLoS Biol. 7:e61. doi: 10.1371/journal.pbio.1000061

Ganea, D. A., Bexter, A., Günther, M., Gardères, P.-M., Kampa, B. M., and Haiss, F. (2020). Pupillary dilations of mice performing a vibrotactile discrimination task reflect task engagement and response confidence. Front. Behav. Neurosci. 14:159. doi: 10.3389/fnbeh.2020.00159

Garcia-Hernandez, N., Bertolotto, F., Cannella, F., Tsagarakis, N. G., and Caldwell, D. G. (2014). How tactor size and density of normal indentation tactile displays affects grating discrimination tasks. IEEE Trans. Haptics 7, 356-366. doi: 10.1109/TOH.2014.2309128

Granholm, E., and Steinhauer, S. R. (2004). Pupillometric measures of cognitive and emotional processes. Int. J. Psychophysiol. 52, 1-6. doi: 10.1016/j.ijpsycho.2003.12.001

Herman, W. X., Smith, R. E., Kronemer, S. I., Watsky, R. E., Chen, W. C., Gober, L. M., et al. (2019). A switch and wave of neuronal activity in the cerebral cortex during the first second of conscious perception. Cerebral Cortex 29, 461-474. doi: $10.1093 /$ cercor/bhx327

Hielscher, E., and Mahar, D. (2017). An exploration of the interaction between touch avoidance and the pleasant touch (C-Tactile Afferent) system. Perception 46, 18-30. doi: 10.1177/0301006616661938

Hoffmann, M., Chinn, L. K., Somogyi, E., Heed, T., Fagard, J., Lockman, J. J., et al. (2017). "Development of reaching to the body in early infancy: From experiments to robotic models," in 2017 Joint IEEE International Conference on Development and Learning and Epigenetic Robotics (ICDL-EpiRob) (Lisbon), 112-119. doi: 10.1109/DEVLRN.2017.8329795

Holmqvist, K., Nyström, M., Andersson, R., Dewhurst, R., Jarodzka, H., and Van De Weijer, J. (2011). Eye Tracking: A Comprehensive Guide to Methods and Measures. Croydon; London: OUP Oxford.

Iriki, A., Tanaka, M., and Iwamura, Y. (1996). Attention-induced neuronal activity in the monkey somatosensory cortex revealed by pupillometrics. Neurosci. Res. 25, 173-181. doi: 10.1016/0168-0102(96)01043-7

Joshi, S., Li, Y., Kalwani, R. M., and Gold, J. I. (2016). Relationships between pupil diameter and neuronal activity in the locus coeruleus, colliculi, and cingulate cortex. Neuron 89, 221-234. doi: 10.1016/j.neuron.2015.11.028

Kang, O., and Wheatley, T. (2015). Pupil dilation patterns reflect the contents of consciousness. Conscious. Cogn. 35, 128-135. doi: 10.1016/j.concog.2015.05.001

Kim, S.-C., Kim, C.-H., Yang, G.-H., Yang, T.-H., Han, B.-K., Kang, S.-C., et al. (2009). "Small and lightweight tactile display (SaLT) and its application," in World Haptics 2009-Third Joint EuroHaptics conference and Symposium on Haptic Interfaces for Virtual Environment and Teleoperator Systems (Salt Lake City, UT: IEEE), 69-74. doi: 10.1109/WHC.2009.4810820

Laeng, B., and Endestad, T. (2012). Bright illusions reduce the eye's pupil. Proc. Natl. Acad. Sci. U.S.A. 109, 2162-2167. doi: 10.1073/pnas.111829 8109

Laeng, B., Sirois, S., and Gredebäck, G. (2012). Pupillometry: a window to the preconscious? Perspect. Psychol. Sci. 79, 18-27. doi: 10.1177/1745691611427305

Lederman, S. J., and Klatzky, R. L. (2009). Haptic perception: a tutorial. Atten. Percep. Psychophys. 71, 1439-1459. doi: 10.3758/APP.71.7. 1439

Lee, C., and Margolis, D. (2016). Pupil dynamics reflect behavioral choice and learning in a Go/NoGo tactile decision-making task in mice. Front. Behav. Neurosci. 10:200. doi: 10.3389/fnbeh.2016.00200

Lee, C. C. Y., Kheradpezhouh, E., Diamond, M. E., and Arabzadeh, E. (2020). State-dependent changes in perception and coding in the mouse somatosensory cortex. Cell Rep. 32:108197. doi: 10.1016/j.celrep.2020.108197
Li, Q., Hill, Z., and He, B. J. (2014). Spatiotemporal dissociation of brain activity underlying subjective awareness, objective performance and confidence. J. Neurosci. 34, 4382-4395. doi: 10.1523/JNEUROSCI.1820-13.2014

Liberati, A., Altman, D. G., Tetzlaff, J., Mulrow, C., Gotzsche, P. C., Ioannidis, J. P., et al. (2009). The PRISMA statement for reporting systematic reviews and meta-analyses of studies that evaluate health care interventions: explanation and elaboration. J. Clin. Epidemiol. 62, e1-e34. doi: 10.1016/j.jclinepi.2009.06.006

Liu, Y., Rodenkirch, C., Moskowitz, N., Schriver, B., and Wang, Q. (2017). Dynamic lateralization of pupil dilation evoked by locus coeruleus activation results from sympathetic, not parasympathetic, contributions. Cell Rep. 20, 3099-3112. doi: 10.1016/j.celrep.2017.08.094

Loken, L. S., Evert, M., and Wessberg, J. (2011). Pleasantness of touch in human glabrous and hairy skin: order effects on affective ratings. Brain Res. 1417, 9-15. doi: 10.1016/j.brainres.2011.08.011

Luna, B., Velanova, K., and Geier, C. F. (2008). Development of eye-movement control. Brain Cogn. 68, 293-308. doi: 10.1016/j.bandc.2008.08.019

Medathati, N. V. K., Desai, R., and Hillis, J. (2020). "Towards inferring cognitive state changes from pupil size variations in real world conditions," in Eye Tracking Research and Applications Symposium (ETRA), eds A. Bulling, A. Huckauf, E. Jain, R. Radacli and D. Weiskopf (Stuttgart: Association for Computing Machinery). doi: 10.1145/3379155.3391319

Michel, M., Beck, D., Block, N., Blumenfeld, H., Brown, R., Carmel, D., et al. (2019). Opportunities and challenges for a maturing science of consciousness. Nat. Human Behav. 3, 104-107. doi: 10.1038/s41562-019-0531-8

Miguel, H. O., Goncalves, O. F., Cruz, S., and Sampaio, A. (2019). Infant brain response to affective and discriminative touch: a longitudinal study using fNIRS. Soc. Neurosci. 14, 571-582. doi: 10.1080/17470919.2018.1536000

Miller, G. (2005). What is the biological basis of consciousness? Science 309:79. doi: 10.1126/science.309.5731.79

Moher, D., Liberati, A., Tetzlaff, J., Altman, D. G., and Group, P. (2009). Preferred reporting items for systematic reviews and meta-analyses: the PRISMA statement. PLoS Med. 6:e1000097. doi: 10.1371/journal.pmed.1000097

Moy, G., Wagner, C., and Fearing, R. S. (2000). "A compliant tactile display for teletaction," in Proceedings 2000 ICRA. Millennium Conference. IEEE International Conference on Robotics and Automation. Symposia Proceedings (Cat. No. 00CH37065) (San Francisco, CA: IEEE), 3409-3415. doi: 10.1109/ROBOT.2000.845247

Mückschel, M., Ziemssen, T., and Beste, C. (2020). Properties of lower level processing modulate the actions of the norepinephrine system during response inhibition. Biol. Psychol. 152:107862. doi: 10.1016/j.biopsycho.2020.107862

Ninomiya, T., Okayama, Y., Matsumoto, Y., Arouette, X., Osawa, K., and Miki, N. (2011). MEMS-based hydraulic displacement amplification mechanism with completely encapsulated liquid. Sensors Actuat. A: Phys. 166, 277-282. doi: 10.1016/j.sna.2009.07.002

Ninomiya, T., Osawa, K., Okayama, Y., Matsumoto, Y., and Miki, N. (2009). "MEMS tactile display with hydraulic displacement amplification mechanism," in 2009 IEEE 22nd International Conference on Micro Electro Mechanical Systems: IEEE, 467-470. doi: 10.1109/MEMSYS.2009.4805420

Partala, T., and Surakka, V. (2003). Pupil size variation as an indication of affective processing. Int. J. Hum. Comput. Stud. 59, 185-198. doi: 10.1016/S1071-5819(03)00017-X

Pins, D., and Ffytche, D. (2003). The neural correlates of conscious vision. Cerebral Cortex 13, 461-474. doi: 10.1093/cercor/13.5.461

Piquado, T., Isaacowitz, D., and Wingfield, A. (2010). Pupillometry as a measure of cognitive effort in younger and older adults. Psychophysiology 47, 560-569. doi: 10.1111/j.1469-8986.2009.00947.x

Pitts, M. A., Metzler, S., and Hillyard, S. A. (2014). Isolating neural correlates of conscious perception from neural correlates of reporting one's perception. Front. Psychol. 5:1078. doi: 10.3389/fpsyg.2014.01078

Ress, D., and Heeger, D. J. (2003). Neuronal correlates of perception in early visual cortex. Nat. Neurosci. 6, 414-420. doi: 10.1038/nn1024

Rigato, S., Banissy, M. J., Romanska, A., Thomas, R., Van Velzen, J., and Bremner, A. J. (2019). Cortical signatures of vicarious tactile experience in four-monthold infants. Dev. Cogn. Neurosci. 35, 75-80. doi: 10.1016/j.den.2017.09.003

Sailer, U., Triscoli, C., Haggblad, G., Hamilton, P., Olausson, H., and Croy, I. (2016). Temporal dynamics of brain activation during 40 minutes of pleasant touch. Neuroimage 139, 360-367. doi: 10.1016/j.neuroimage.2016.06.031 
Sarakoglou, I., Tsagarakis, N. G., and Caldwell, D. G. (2012). "A compact tactile display suitable for integration in VR and teleoperation," in 2012 IEEE International Conference on Robotics and Automation (Saint Paul, MN: IEEE), 1018-1024. doi: 10.1109/ICRA.2012.6225248

Sarakoglou, I., Tsagarakis, N. G., and Caldwell, D. G. (2014). "Development of a hybrid actuator with controllable mechanical damping," in 2014 IEEE International Conference on Robotics and Automation (ICRA) (Hong Kong: IEEE), 1078-1083. doi: 10.1109/ICRA.2014.6906988

Schneider, E. R., Gracheva, E. O., and Bagriantsev, S. N. (2016). Evolutionary specialization of tactile perception in vertebrates. Physiology 31, 193-200. doi: 10.1152/physiol.000 36.2015

Schriver, B. J., Bagdasarov, S., and Wang, Q. (2018). Pupil-linked arousal modulates behavior in rats performing a whisker deflection direction discrimination task. J. Neurophysiol. 120, 1655-1670. doi: 10.1152/jn.00290.2018

Schriver, B. J., Perkins, S. M., Sajda, P., and Wang, Q. (2020). Interplay between components of pupil-linked phasic arousal and its role in driving behavioral choice in Go/No-Go perceptual decision-making. Psychophysiology 57:e13565. doi: 10.1111/psyp.13565

Sirois, S., and Brisson, J. (2014). Pupillometry. Wiley Interdisc. Rev. 5, 679-692. doi: $10.1002 /$ wcs. 1323

Streque, J., Talbi, A., Pernod, P., and Preobrazhensky, V. (2012). Pulsedriven magnetostatic micro-actuator array based on ultrasoft elastomeric membranes for active surface applications. J. Micromech. Microeng. 22:095020. doi: 10.1088/0960-1317/22/9/095020

Tatler, B. W., Kirtley, C., Macdonald, R. G., Mitchell, K. M., and Savage, S. W. (2014). "The active eye: Perspectives on eye movement research," in Current Trends in Eye Tracking Research, eds M. Horsley, M. Eliot, B. A., Knight and R. Reilly (Cham: Springer), 3-16. doi: 10.1007/978-3-319-02868-2_1

Unesco (2005). Universal Declaration on Bioethics and Human Rights. Available online at: https://en.unesco.org/themes/ethics-science-and-technology/ bioethics-and-human-rights (accessed December 28, 2020).

Van Hooijdonk, R., Mathot, S., Schat, E., Spencer, H., Van Der Stigchel, S., and Dijkerman, H. C. (2019). Touch-induced pupil size reflects stimulus intensity, not subjective pleasantness. Experi. Brain Res. 237, 201-210. doi: 10.1007/s00221-018-5404-2
Van Kempen, J., Loughnane, G. M., Newman, D. P., Kelly, S. P., Thiele, A., O'connell, R. G., et al. (2019). Behavioural and neural signatures of perceptual decision-making are modulated by pupil-linked arousal. Elife 8:e42541. doi: 10.7554/eLife.42541.001

Velázquez, R., Pissaloux, E. E., Hafez, M., and Szewczyk, J. (2008). Tactile rendering with shape-memory-alloy pin-matrix. IEEE Trans. Instrum. Meas. 57, 1051-1057. doi: 10.1109/TIM.2007.913768

Velázquez, R., Pissaloux, E. E., and Wiertlewski, M. (2006). “A compact tactile display for the blind with shape memory alloys," in Proceedings 2006 IEEE International Conference on Robotics and Automation, 2006. ICRA 2006 (Orlando, FL), 3905-3910. doi: 10.1109/ROBOT.2006.1642300

Wetzel, N., Buttelmann, D., Schieler, A., and Widmann, A. (2016). Infant and adult pupil dilation in response to unexpected sounds. Dev. Psychobiol. 58, 382-392. doi: $10.1002 /$ dev.21377

Wyart, V., and Tallon-Baudry, C. (2008). Neural dissociation between visual awareness and spatial attention. J. Neurosci. 28, 2667-2679. doi: 10.1523/JNEUROSCI.4748-07.2008

Yang, H., Bari, B. A., Cohen, J. Y., and O'connor, D. H. (2020). Locus coeruleus spiking differently correlates with somatosensory cortex activity and pupil diameter. bioRxiv [Preprint]. doi: 10.1101/2020.06.04.134676

Yoo, J., Yun, S., Lim, S.-C., Park, J., Yun, K.-S., and Lee, H.-K. (2015). Position controlled pneumatic tactile display for tangential stimulation of a finger pad. Sensors Actuators A: Phys. 229, 15-22. doi: 10.1016/j.sna.2015. 03.023

Conflict of Interest: The authors declare that the research was conducted in the absence of any commercial or financial relationships that could be construed as a potential conflict of interest.

Copyright (c) 2021 Gusso, Serur and Nohama. This is an open-access article distributed under the terms of the Creative Commons Attribution License (CC BY). The use, distribution or reproduction in other forums is permitted, provided the original author(s) and the copyright owner(s) are credited and that the original publication in this journal is cited, in accordance with accepted academic practice. No use, distribution or reproduction is permitted which does not comply with these terms. 